\title{
The effect of time on the evolution of the martensite structure and strain caused by magnetic field in $\mathrm{Ni}_{2} \mathrm{MnGa}$ shape memory alloys
}

\author{
N. Glavatska, V. Gavriljuk, I. Glavatsky, Y. Ge ${ }^{1}$, O. Söderberg ${ }^{1}$, A. Jääskeläinen ${ }^{1}$, \\ K. Ullakko ${ }^{2}$ and V.K. Lindroos ${ }^{1}$
}

Institute for Metal Physics, Department of Alloyed Steels, $003680 \mathrm{Kiev} 142$, Ukraine

${ }^{1}$ Helsinki University of Technology, Laboratory of Physical Metallurgy and Materials Science, P.O. Box 6200, 02015 Hut, Finland

${ }^{2}$ Helsinki University of Technology, Laboratory of Biomedical Engineering, P.O. Box 2200, 02015 Hut, Finland

\begin{abstract}
Kinetics of the martensitic transformation, change in the preferential martensite variants, field-induced strain and magnetisation under applied magnetic field are studied in-situ. Two $\mathrm{Ni}_{2} \mathrm{MnGa}$ alloys of the nonstoichiometric composition which reveal large strain $(0,5-5 \%)$ under magnetic field were investigated using the $\mathrm{X}$ ray diffraction $\theta-2 \theta$ analysis, optical metallography and measurements of the field-induced strain. It is shown that evolution of the martensitic structure under applied magnetic field occurs with time at constant values of the field and temperature. The kinetics of the direct and inverse processes is strongly determined by the relation between the crystallographic direction of easy magnetisation and that of the applied magnetic field. The results of the X-Ray diffraction study are consistent with the data of optical microscopy and measurements of the field-induced strain.
\end{abstract}

\section{INTRODUCTION}

Magnetically controlled strain (magnetic shape memory effect, MSME) in Ni-Mn-Ga alloys is based on the reorientation of the twin structure of martensite under applied magnetic field [1-3]. A mechanism of the magnetic shape memory effect presented firstly in [1,2] includes the magnetically induced motion of the martensite twin boundaries. As these alloys are expected to exhibit a great potential as actuators and sensors, it is important to study their behavior under applied magnetic field. Effect of the applied magnetic field was revealed in the X-ray study of the polycrystalline Ni-Mn-Ga alloy as a change of intensity of the martensite variants [4]. A strong magnetic anisotropy is shown to be a necessary condition to provide sufficient magnetic pressure for the movement of the twin boundaries resulting in the large field-induced strain [5]. On the other hand the time effects are known in magnetization [6]. Therefore studies of the kinetics of magnetically controlled change in the martensitic structure seem to be important. Firstly the time effects in the direct and inverse processes of the martensitic transformation and the fieldinduced strain caused by the magnetic field in the Ni-Mn-Ga alloy were found in [7]. The aim of this study is to clarify an effect of time on the large field-induced strain and on the evolution of the martensitic structure in $\mathrm{Ni}_{2} \mathrm{MnGa}$ alloys under applied magnetic field and after magnetic field removal.

\section{EXPERIMENTAL}

Two ingots $\mathbf{A}(49.6 \mathrm{Ni}, 28.4 \mathrm{Mn}, 22 \mathrm{Ga}$ in at.\%) and $\mathbf{B}(49.7 \mathrm{Ni}, 28.7 \mathrm{Mn}, 21.6 \mathrm{Ga}$ in at.\%) of the nonstoichiometric $\mathrm{Ni}_{2} \mathrm{MnGa}$ composition were homogenized for $72 \mathrm{~h}$ at $1273 \mathrm{~K}$. Curie point $\mathrm{T}_{\mathrm{C}}=95^{\circ} \mathrm{C}$ and the temperatures of martensitic transformations $\mathrm{M}_{\mathrm{s}}=33^{\circ} \mathrm{C}, \mathrm{M}_{\mathrm{f}}=31^{\circ} \mathrm{C}, \mathrm{A}_{\mathrm{s}}=37^{\circ} \mathrm{C}, \mathrm{A}_{\mathrm{f}}=40^{\circ} \mathrm{C}$ for the first alloy and $\mathrm{M}_{\mathrm{s}}=32{ }^{\circ} \mathrm{C}, \mathrm{M}_{\mathrm{f}}=29^{\circ} \mathrm{C}, \mathrm{A}_{\mathrm{s}}=38^{\circ} \mathrm{C}, \mathrm{A}_{\mathrm{f}}=41^{\circ} \mathrm{C}$ for the second one were determined from the temperature dependence of the low field magnetic susceptibility. The polycrystalline ingot $\mathbf{A}$ had the texture $<100\rangle$ along the direction of crystallization and was cut into pieces of the size of $2 \times 5 \times 10 \mathrm{~mm}^{3}$ parallel and orthogonal to this axis for specimens $\mathbf{A 1}$ and $\mathbf{A 2}$, respectively. The single crystalline specimen $\mathbf{B}\left(4 \times 7 \times 10 \mathrm{~mm}^{3}\right)$ was cut from some big grain of the ingot $\mathbf{B}$ so that its surfaces were orthogonal or parallel to the direction of crystallization $\langle 100\rangle$. All specimens were ground and electropolished in 25 $\% \mathrm{HNO}_{3}$ - ethanol electrolyte at ambient temperature.

The solution treated polycrystalline specimens did not reveal strong magnetic anisotropy. Anisotropy was induced by a magneto-thermo-mechanical treatment (MTM). During this treatment specimens were heated above of $\mathrm{M}_{\mathrm{s}}$ temperature and then cooled slowly to the ambient temperature under magnetic field 
of 0,25 to $0,4 \mathrm{~T}$ and a compressive stress applied parallel to [110] for specimen A1, [100] for specimen A2 and [001] for specimen B1. Direction of the magnetic field was the same as that of compression.

The X-ray diffraction was measured without rotation of the sample using Philips diffractometer PW1718 equipped by monochromator. $\mathrm{Cu}_{\mathrm{K \alpha}}$ radiation was used. The sample was clamped to the special specimen holder so that its position remained unchanged during the measurements. Magnetic field during $\mathrm{X}$-Ray diffraction measurements was produced by Fe-Nd-B permanent magnets of $0,25 \mathrm{~T}, 0,3 \mathrm{~T}$ and $0,4 \mathrm{~T}$ adjusted on the holder and fixed to the goniometer axis. Motion of the martensite twins produced by the magnetic field caused the change in the relative intensity of the different martensite variants studied by Xray theta-two theta analysis.

The field-induced strain was measured using a load cell equipped with a capacitive sensor or strain gages fixed at the samples if those showed small strain under magnetic field. The magnetic field of up to $1,2 \mathrm{~T}$ was applied parallel or orthogonal to the direction of easy magnetization. Optical microscopy studies were carried out at room temperature using the inverted research microscope Olympus PMG3.

\section{RESULTS AND DISCUSSION}

Effect of the time exposures on the field-induced strain is illustrated in Fig 1. It is seen that the value and the sign of the strain depend on the preliminary treatment.
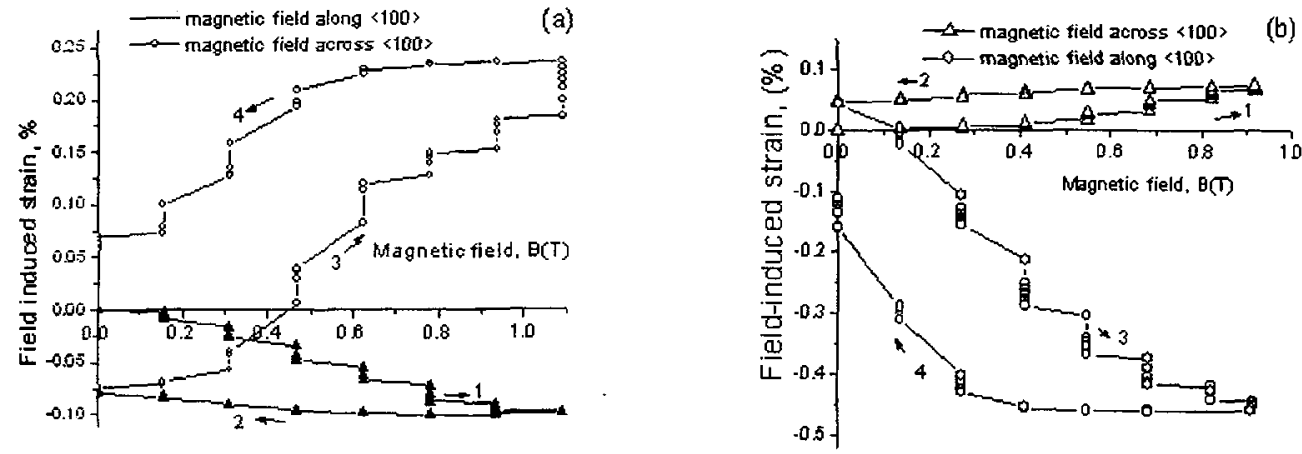

(c)

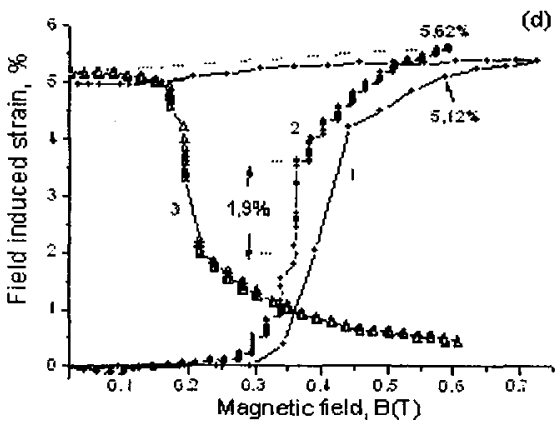

Figure 1: Field and time dependence of field-induced strain: (a) specimen $\mathbf{A} 2$ before measurements was magnetized using MTM close to direction [001], stress along [001], (b) specimen A2, after the procedure described in (a) it was magnetized using MTM by field $0.25 \mathrm{~T}$ - along [001], stress - across [001], (c) specimen $\mathbf{A 2}$, after the procedures (a+b) it was magnetized using MTM by field $0.4 \mathrm{~T}$ along [001] under stress - across [001], before the measurement the sample was magnetized along [100], (d) - single crystal specimen B1, (1,2)- field across [100], (3) - field along [100]. The time exposure is equal to $5 \mathrm{~min}$ (a), (b), (c) and $1 \mathrm{sec}$ for (1) and $27 \mathrm{sec}$ for $(2,3)$. The numbers 1-4 mean the order of measurements.

The direction of easy magnetisation close to [001] was induced under field of $0.25 \mathrm{~T}$ for the specimen in Fig. 1a. This sample did not show any definite direction of possible magnetisation. The time effect (direct and inverse) is quite small if the magnetic field was applied close to the direction of easy magnetisation $(1,2)$, however, it is remarkable for other direction $(3,4)$ at all values of the field. 
After this procedure the specimen $\mathbf{A 2}$ had undergone the MTM treatment under magnetic field of $0,25 \mathrm{~T}$ producing [001] preferential martensite variant, but the mechanical stress was applied orthogonal to magnetic field (Fig.1 b). The strain under magnetic field applied in the direction of the preliminary magnetization is rather small whereas the application of the field in the orthogonal direction results in increased strain.

A more homogeneous crystallographic and magnetic structure was produced when, after procedures $(\mathbf{a}+\mathbf{b})$, a larger field was again applied along direction [001] with the mechanical loading across [001] during MTM treatment. The direction of easy magnetisation was produced for specimen $\mathbf{A 2}$ along [001] and an orthogonal direction was found as possible direction of magnetisation. The sample was magnetized along [1.00] before measurements. As a result, a larger field-induced strain was obtained (Fig. 1c). The time effect was observed in this case mainly under field close to $0.4 \mathrm{~T}$.

For the single crystal B1 both directions of easy and possible magnetization were induced by MTM. Figure 1d shows the largest field-induced strain produced by the change from the possible to easy direction of magnetization corresponding to [100] and [001], respectively. It was found that increase in the time of exposures under constant values of the magnetic field increases the field-induced strain (Fig. 1d, curves 2,3). This increase takes place under the field of 0,2-0,4T. Furthermore, the direction of the applied field affects the time dependence of strain. Let us note that the time dependence of the strain is larger under increased magnetic field, i.e. during direct process (Fig.1b,c,d). To clarify effect of the time exposure on the field-induced strain, the X-ray diffraction study was performed.
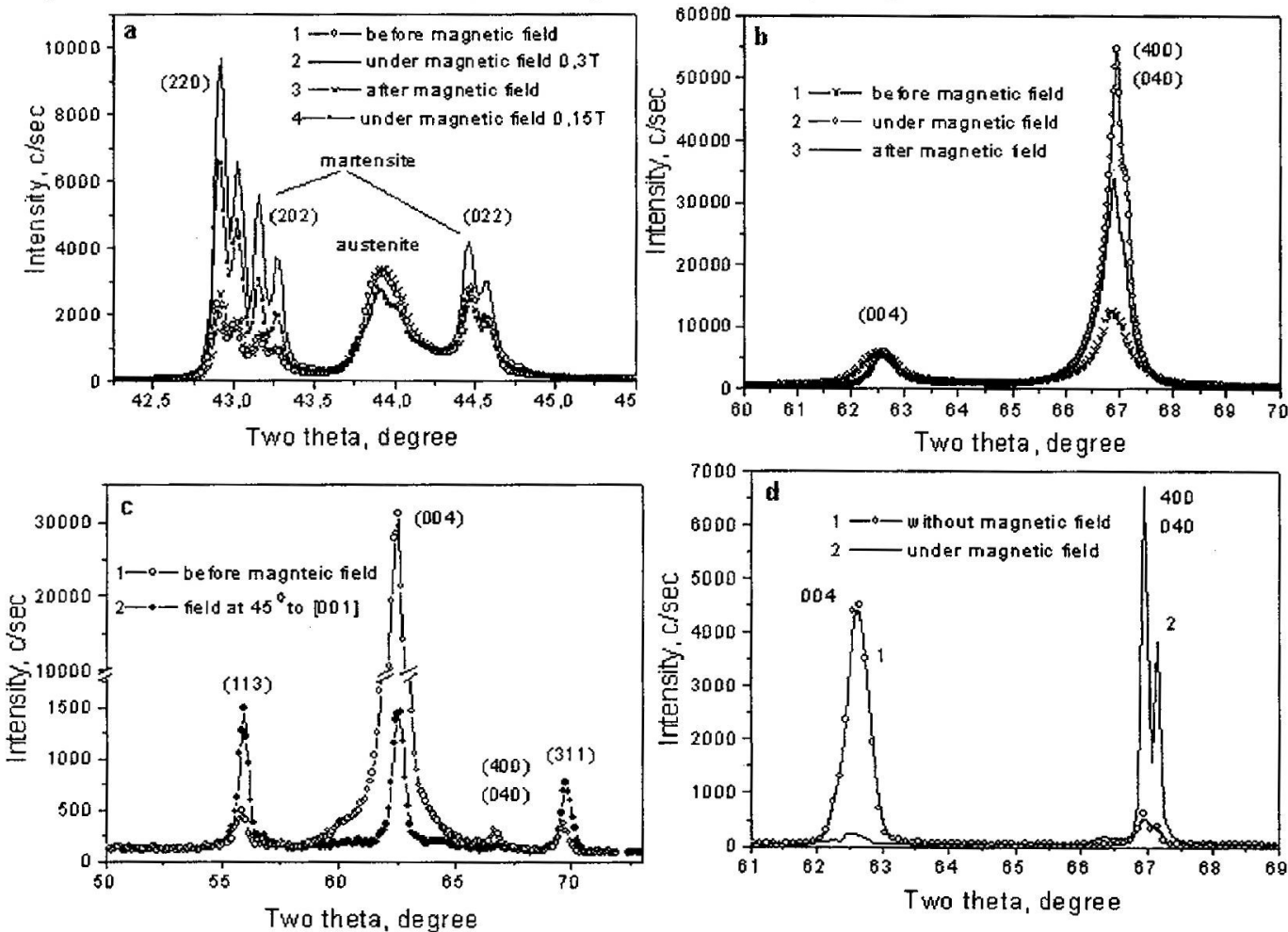

Figure 2: Effect of the magnetic field direction: a - specimen A1, martensite and austenite, magnetic field of $B=0.25 T$ is applied orthogonal to the studied surface, i.e. parallel to direction $\langle 110\rangle$; $\mathbf{b}$ - specimen $\mathbf{A 2}$, martensite, magnetic field of $0,25 T$ is applied parallel to direction [100]; c- specimen A2, martensite, magnetic field of $\mathrm{B}=0.4 \mathrm{~T}$ is applied at $45^{\circ}$ to the surface; $\mathbf{d}$ specimen B1, martensite, (1) after MTM and preliminary magnetization; (2) preferential (400), (040) orientation is induced under magnetic field applied orthogonal to the surface with preliminary orientation (001): (1)- before, (2)- under magnetic field 

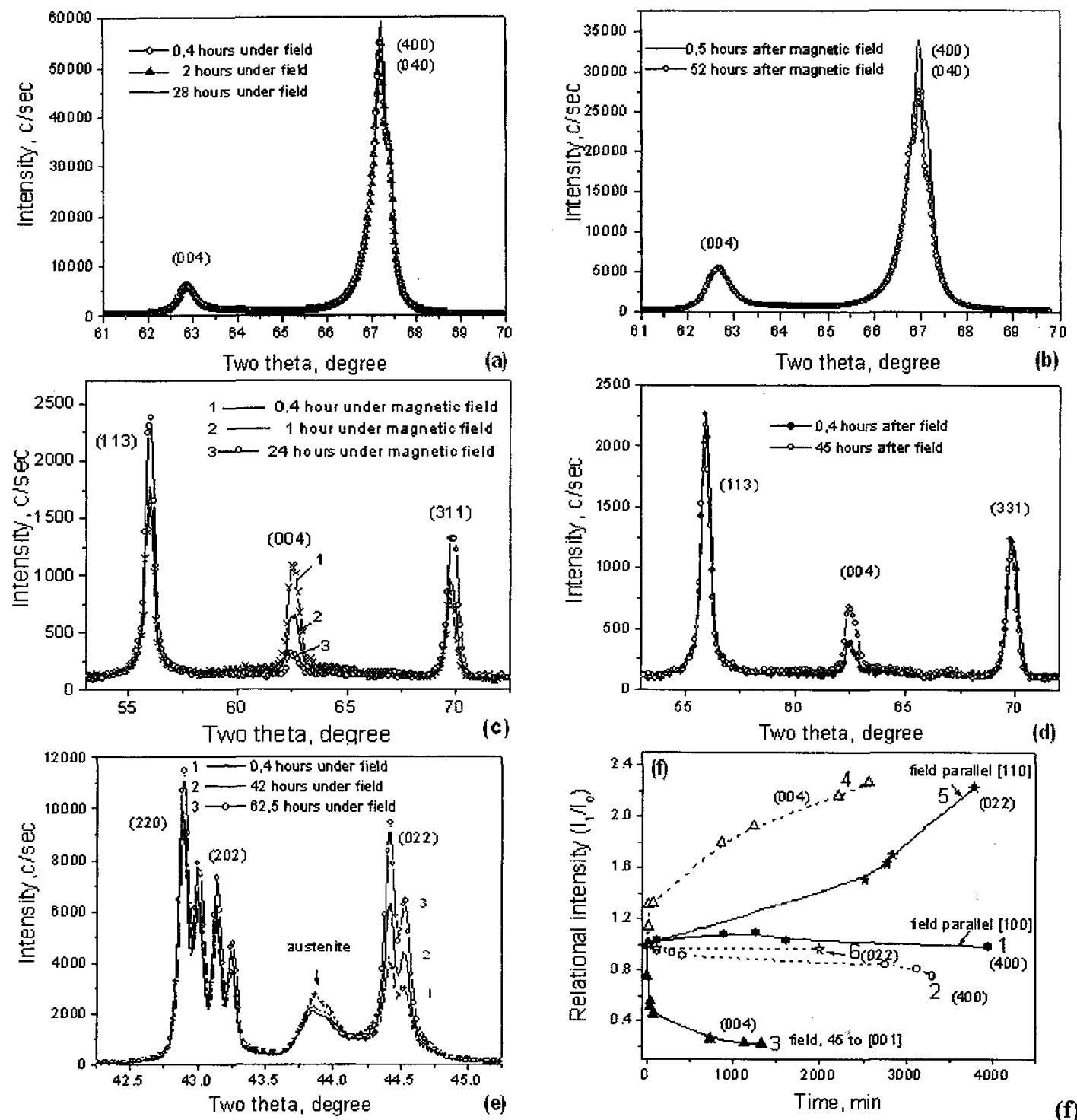

(f)

Figure 3: Effect of the exposure under the magnetic field 0,3T on the intensity of X-Ray diffraction: (a) the specimen A2 under magnetic field applied along [100]; (b) specimen A2, magnetic field applied along [100] is taken out; (c) specimen $\mathbf{A 2}$ under magnetic field applied at $45^{\circ}$ to [001]; (d) specimen $\mathbf{A 2}$, magnetic field applied at $45^{\circ}$ to [001] is taken out; (e) the specimen A1 under magnetic field applied along $\langle 110\rangle$; (f) effect of the field direction on time dependence of relational intensity. Solid lines - under magnetic field, dotted lines - after magnetic field is taken out.

A segment of the diffraction pattern for specimen $\mathbf{A 1}$ before application of the magnetic field is shown in Fig. 2a (curve 1). After the magnetic field was applied parallel to $\langle 110\rangle$ direction, the intensity of the martensite reflections was strongly increased and the intensity of the austenitic reflection decreased (Fig.1a, curve 2). After the magnet was taken out, all the peaks restored their positions and acquired practically the same intensity as before applying the magnetic field (Fig.2a, curve 3).

For specimen A2 the direction of easy magnetisation was induced parallel to direction [100] (Fig.2b, curve1). For this specimen the diffraction pattern is not restored after taking out the field if the magnetic field was applied along the axis of easy magnetisation (Fig. 2b, curve 3). The situation is different if the 
magnetic field was applied at $45^{\circ}$ to the direction of easy magnetisation (Fig.2c). In this case the orientation of the polycrystalline specimen was completely changed under magnetic field (Fig. 2c, curve 2 ) and it is not restored after the field was taken out (Fig.2c, curve 3). The preferential (001) orientation of martensite was induced in specimen B1 during MSM measurements by magnetization under field of 1 $\mathrm{T}$ (Fig. 2d, (1)). As follows from Fig. 2d, (2), the magnetic field applied along direction [001] has caused a total redistribution of the intensities of the martensite variants, so that the preferential orientation (001) of martensite was converted to (100). It means, that the motion of martensite twins has caused a reorientation of the crystal lattice, so that the axis of easy magnetisation is oriented along direction [100]. This orientation remains unchanged after the magnetic field is taken out.

Thus, obtained results argue that the direction of the applied magnetic field strongly affects the martensite structure causing a change in the preferential orientation of the martensite.

X-ray diffraction was also used in the study of time-dependent effects. As follows from Fig. 3 a,c,e,f, the evolution of the martensite structure occurs under the fixed value of the magnetic field during the time exposure and this process strongly depends on the direction of the applied field. If the field is applied close to the axis of easy magnetisation, the intensity of reflection (400) remains practically unchanged with time (Fig. $3 \mathrm{a}, \mathrm{f}$ ). If the magnetic field is applied at $45^{\circ}$ to direction [001], the intensity of the martensite variant (001) is decreased abruptly at the start of exposure and then changes slowly (Fig. 3c,f). If the field is applied orthogonal to the direction of easy magnetisation and along [110], the intensity of the martensite reflections (022) is increased permanently during exposures (Fig. 3e,f). The change in the intensity of X-Ray diffraction reflects the growth or disappearance of corresponding martensite variants caused by the field-induced movement of the twin boundaries.

The evolution of the martensitic structure with time after magnetic field was taken out is shown in Fig. $3 b$,d,f. If the magnetic field was applied close to the direction $<001\rangle$, the magnetically induced increase in the intensity of reflection (400) diminishes with time after the action of the field is over (Fig.3b,f curve 2) and does not approach the initial state. Reflection (004) increases permanently in intensity after the field applied at $45^{\circ}$ to direction [001] is taken out (Fig. 3d,f curve 4). Nevertheless, the initial intensity is not restored. If the magnetic field was close to direction $\langle 110\rangle$, the magnetically induced change in the martensitic structure disappears quickly to the initial state and thereafter practically nothing changes with time (Fig. 3f, curve 6).

Thus, the crystallographic orientation of martensite is changed with time depending on the relation between the direction of easy magnetization and that of the applied magnetic field. If the magnetic field is applied along the axis of easy magnetization, the crystallographic structure changes quickly under magnetic field whereas the inverse change in the absence of field occurs slowly. In contrast, in the case of the unfavorable axis of magnetization, the change in the crystallographic structure occurs slowly with time under magnetic field and restores quickly after magnetic field is taken out.

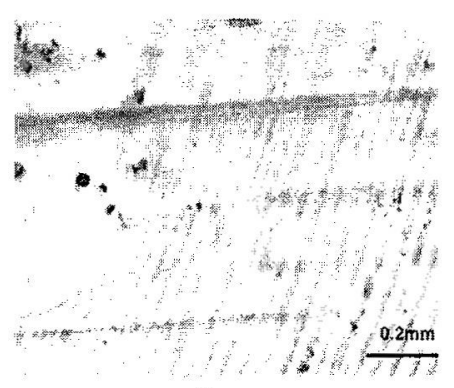

$\mathbf{a}$

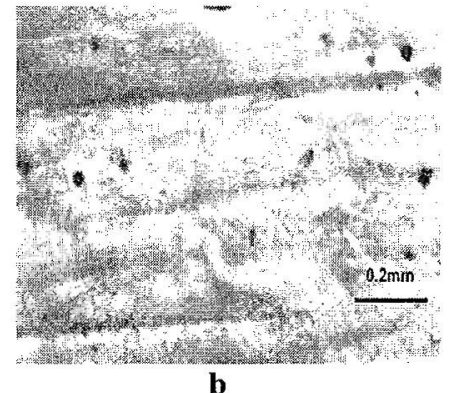

b

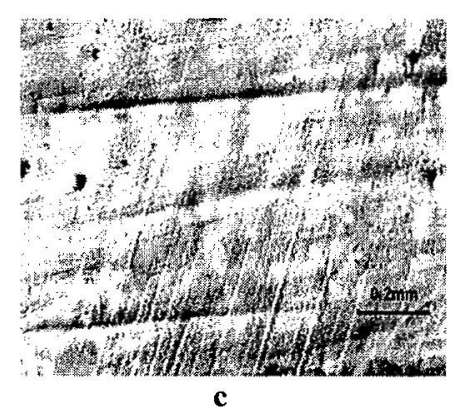

c

Figure 4: After-effect of the magnetic field on the martensitic structure: the specimen $\mathbf{B}$, (a) magnetic field was applied along $<100>$ and taken out, (b) thereafter magnetic field was applied along $<110>, 1 \mathrm{~min}$ after it was taken out, (c) the same as (b), exposure $100 \mathrm{~min}$. The studied surface is orthogonal to direction $<100>$.

Optical microscopy studies confirm this conclusion (Fig. 4). The twin martensitic structure is stable with time if the field was applied along the direction of easy magnetization (Fig. 4a), it is completely changed 
if the field was applied in the orthogonal direction (Fig. 4b), however the previous structure of Fig. 4a is restored with time-after this field was taken out (Fig. 4c). The same conclusion follows from Fig. $3 \mathrm{~d}$ that shows increase in the intensity of (004) reflection after magnetic field is taken out.
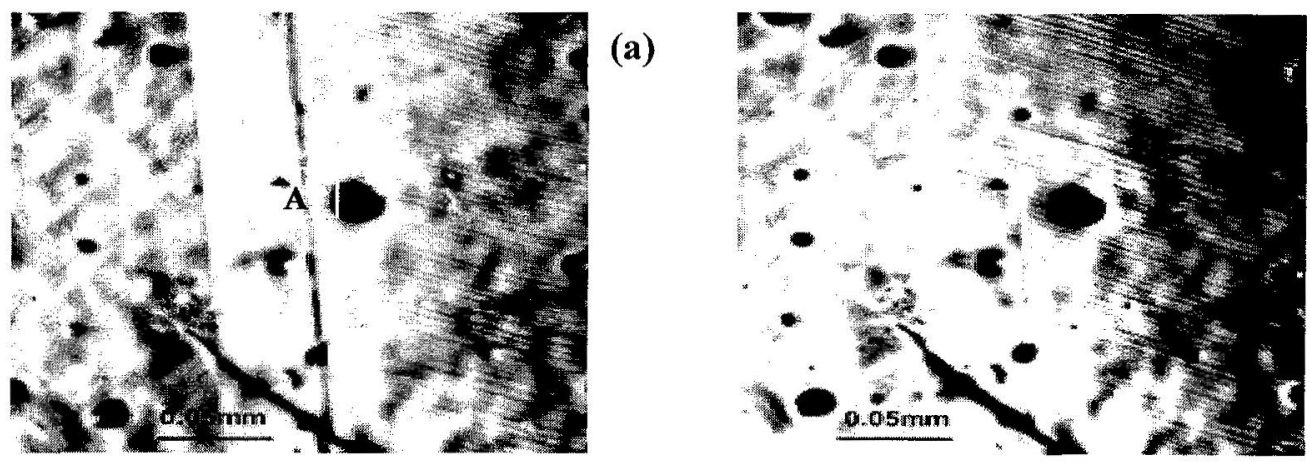

Figure 5: The evolution of the martensite structure of the specimen $\mathbf{B}$ under magnetic field $0,3 \mathrm{~T}$, the direction of the field is normal to the studied surface and normal to the direction of easy magnetization:

(a) $-0,37$ hours under magnetic field, (b) - 17,37 hours under magnetic field

The behavior of the martensitic structure under magnetic field is demonstrated in Fig. 5. If the direction of the applied magnetic field is not consistent with that of easy magnetization, the martensitic structure is developed with time under a constant value of the field. The twin marked by its right boundary A (Fig.5a) disappears with time (Fig.5b) and is being substituted by the thin twins from the right area.

\section{Summary}

A change in the martensitic structure and in the corresponding field-induced strain of the alloys $\mathrm{Ni}_{2} \mathrm{MnGa}$ under applied magnetic field and after the magnetic field is taken out is developed with time at constant values of temperature and field.

The direction of magnetic field strongly affects the change in the martensitic structure and fieldinduced strain and their time dependence.

Magnetically induced structure of martensite is more stable with time after magnetic field is taken out if that was applied in the direction of easy magnetisation.

\section{Acknowledgements}

The financial support of Technology Development Center (TEKES), of Academy of Finland and of the project INTAS-97-30921 is greatly appreciated.

\section{References}

[1] K.Ullakko, J.K.Huang, C.Kantner, R.C.Handley, V.V.Kokorin, Appl. Phys. Lett., 69(13), (1996), pp.1966-1968

[2] K.Ullakko, J. Materials Engineering and Performance, 5, (1996), pp. $405-409$

[3] R.C.Handley, J. Appl. Phys. 83 (6), (1998), pp. 3263-3270

[4] Y. Ezer, A. Sozinov, G. Kimmel, V. Eteläniemi, N.I. Glavatskaya, A. D’Anci, V. Podgursky, V.K. Lindroos and K. Ullakko, Proceedings of SPIE Conference on Smart Materials Technologies, Newport Beach.,California, March 1999, p. 244.

[5] R.D.James and K.F.Hane, Acta mater. 48, (2000), pp. 197-222

[6] G.W.Rathenau, Time Effects in Magnetisation, In: Magnetic Properties of Metals and Alloys, American Society for Metals, Cleveland, (1959), pp.168-199

[7] N.I.Glavatska, K.Ullakko, JMMM, accepted for publication (2000). 